\title{
IMPROVED HYBRID CIRCUIT ASSEMBLY YIELDS AND RELIABILITY BY GLASSIVATION OF THE SEMICONDUCTOR CHIP
}

\author{
B. C. HEAP and S. A. FRANCE \\ Ferranti Limited, Chadderton, England
}

(Received March 5, 1977)

\begin{abstract}
Lead glass passivation (glassivation) of semiconductor chips has been investigated. Glassivation of BC107 type transistor chips has resulted in improved robustness and their ability to withstand ionic contamination better than control chips. Increased yields were observed when glassivated transistor chips were assembled in ceramic flat packages.
\end{abstract}

\section{SUMMARY}

The paper describes the evaluation of lead glass layers deposited from colloidal solutions to form continuous glasses over metallised bipolar transistor chips. BC107 transistors were selected as the test vehicle because of their severe, low current gain specification and the susceptibility of this parameter to ionic contamination, mechanical stress and general mishandling during the assembly process.

Three fundamental properties of the glass layer were investigated:-

1) Thickness (i.e. whether sufficiently thick layers can be deposited before crazing occurs as with silane deposited oxides). The effectiveness of the thick glass layers were evaluated using epoxy encapsulants known to polarise under elevated temperature and bias tests. Results show that compared to standard 'nonglassivated' chips large improvements in the stability of low current gain are made.

2) The effectiveness of the layer as an ionic barrier was evaluated by soaking non-encapsulated glassivated and standard chips in sodium-chloride solution. The devices were then subjected to elevated temperature and bias tests. The results indicate a greatly improved barrier effectiveness.

3) The ability of glassivated devices to withstand various temperature time combinations was evaluated and compared to standard chips. These temperature time combinations were typical of what is experienced in Si Au eutectic die attach sequences and Cerdip/ Cerpak glass frit and sealing operations. The compari- son was made by measuring initial and final low current gain on the 'glassivated' and standard devices and results indicate a greatly improved robustness.

\section{INTRODUCTION}

Standard planar diffused transistors are assembled in hybrid microcircuits by either conductive epoxy or silicon-gold ( $\mathrm{Si}-\mathrm{Au})$ eutectic die attach techniques. Of the two techniques, conductive epoxy is the most generally used because the temperatures involved in die attach are low compared to $\mathrm{Si}-\mathrm{Au}$ eutectic techniques. It is generally acknowledged that as silicon bipolar transistors are subjected to progressively higher temperatures and/or longer times, the probability of parameter degradation is increased.

However for some applications epoxy die attach techniques are not suitable (i.e. low thermal impedance or low $\mathrm{V}_{\mathrm{CE}(\mathrm{SAT})}$ requirements). High reliability may be required where parameter degradation cannot be tolerated when subjecting the circuits to stringent environmental tests. The general silicon transistor or integrated circuit chip is protected or passivated by a layer of $\mathrm{SiO}_{2}$ which affords a great deal of protection. However, there are several ionic species, the most significant of which is sodium (Na+) which becomes mobile in $\mathrm{SiO}_{2}$ at temperatures approaching $150^{\circ} \mathrm{C}$. Consequently if the silicon chip is subjected to temperatures in excess of $150^{\circ} \mathrm{C}$ during the assembly process or in subsequent environmental tests, then the $\mathrm{SiO}_{2}$ affords little protection and parameter degradation is likely. 


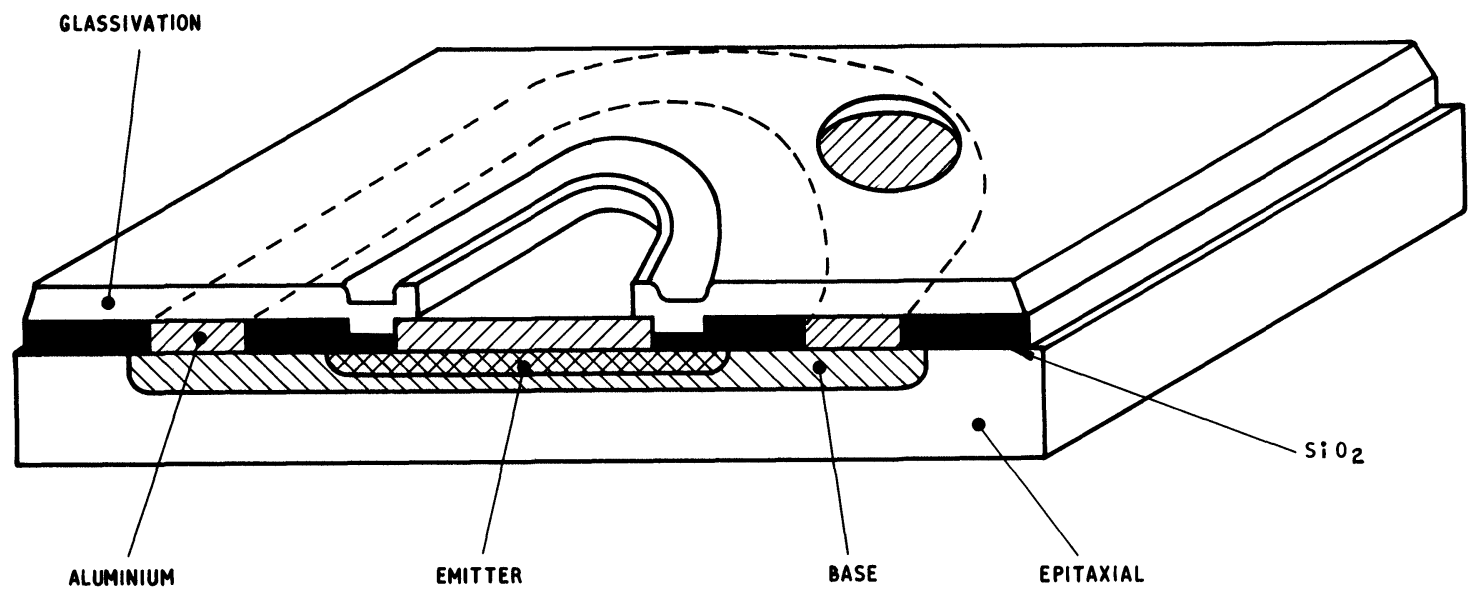

FIGURE 1 Glassivated ZT930 chip.

FAILURES $\%$

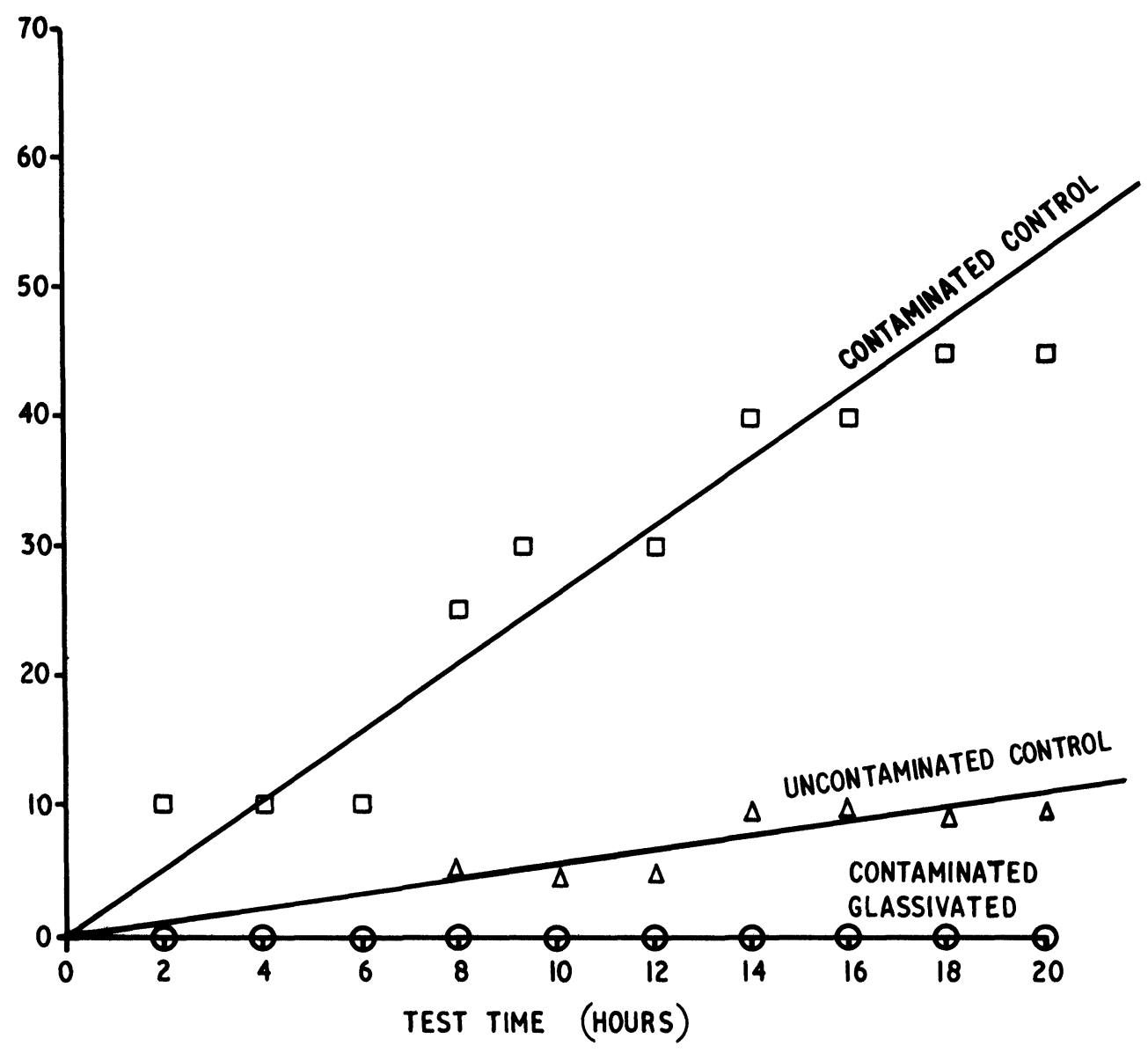

FIGURE 2 Graph of percent failures against test time for contaminated glassed transistors, contaminated control and uncontaminated control in ion getter experiment. 
A solution to the problem is to deposit a glass passivating layer after metallisation and etch contact holes through the glass to the aluminium metallisation to allow subsequent wire bonding. The glass will work effectively as a barrier to ionic contamination and hence decrease the temperature/time susceptibility of the device and also improve its capability of surviving stringent environmental tests.

\section{DISCUSSION}

\subsection{Deposition and Formation of Glass Passivating Layers}

This is done by a sedimentation technique. Glass frit is mixed with methanol and particles of greater than 1 micron diameter are settled out by controlled centrifuging. The methanol left containing grains of 1 micron diameter and less, now called the slurry, is decanted off. Settling agents are added to the slurry such that the dielectric constant of the medium is lowered sufficiently to allow particles to come out of suspension on centrifuging onto an underlying $\mathrm{Si}$ wafer. Too high a dielectric constant results in the lifting of the glass frit after centrifuge and too low a dielectric constant presents localised agglomerates of particles giving non uniform layers. The volume of slurry used per slice is selected so as to deposit a glassy layer $2 \pm .02$ micron thick when fired. The firing temperature of the glass is $550^{\circ} \mathrm{C}$. Standard photoresist techniques are used to open windows in the glass layer to allow wire bonding to the underlaying aluminium metallisation.

An example of a glass passivated transistor is shown in Figure 1.

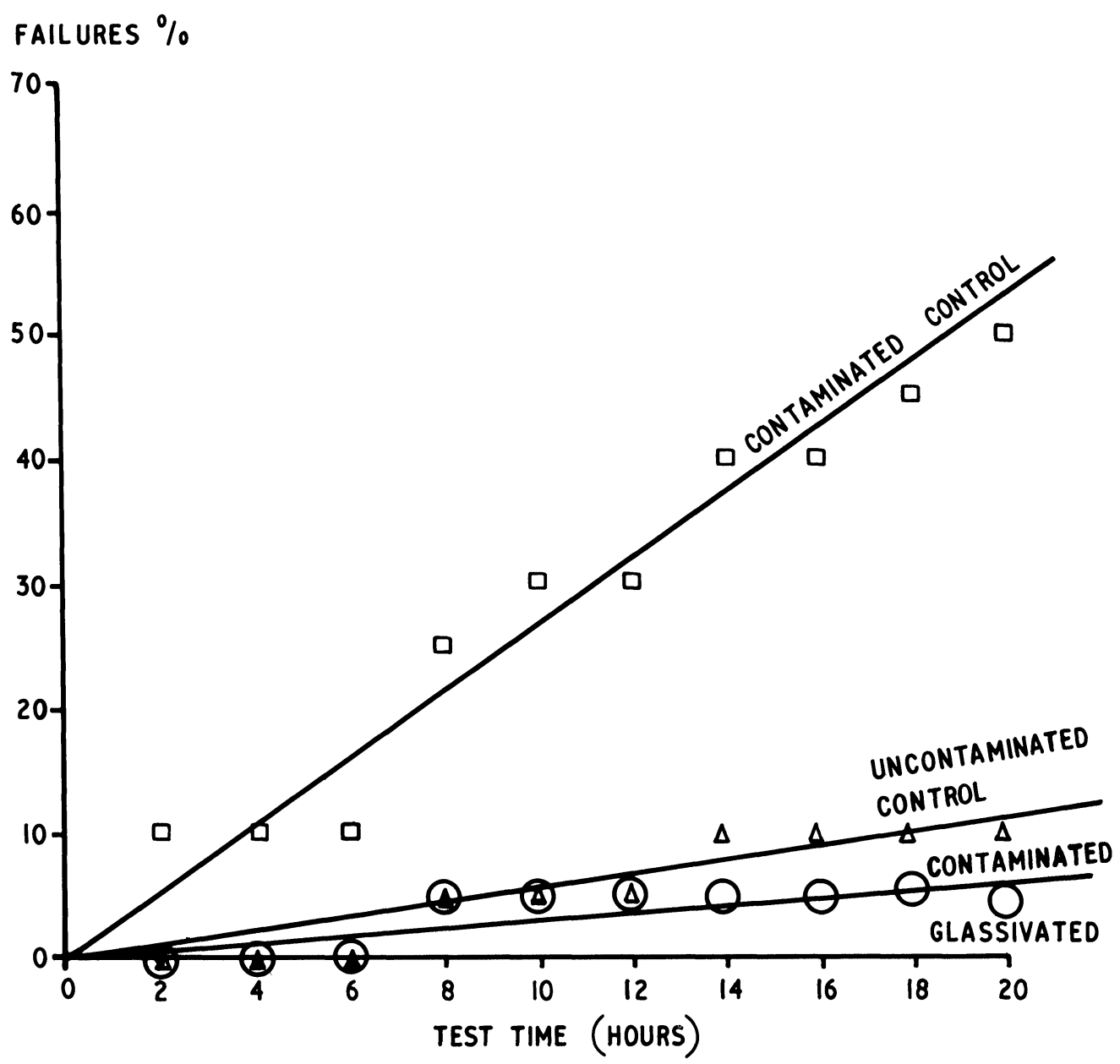

FIGURE 3 Graph of percent failures against test time for contaminated glassed transistors, contaminated control and uncontaminated control in ion barrier experiment. 


\subsection{Gettering Properties of Glassivation}

Three groups of $\mathrm{BC} 107$ transistor chips were processed to evaluate the gettering properties of the glassivation process (i.e. how the firing sequence of the glassivation process mops up any contamination which may be present in the $\mathrm{SiO}_{2}$ passivation layer prior to glass deposition).

Contamination was introduced by taking devices and subjecting them to a $10 \mathrm{~min}$. boil in a 0.02 molar $\mathrm{NaCl}$ solution followed by a bake at $300^{\circ} \mathrm{C}$ for 30 mins. $\mathrm{NaCl}$ solution was chosen because sodium is the most significant ionic contaminant.

A control sample received no $\mathrm{NaCl}$ contamination. A contaminated control sample was processed as described. A contaminated glassivated sample was processed as described and then glassivated. The three groups were assembled in TO18 packages and tested by applying $30 \mathrm{~V}$ reverse bias to the collector-base junction at $165^{\circ} \mathrm{C}$. Figure 2 shows the $\%$ failure of the devices against test time. A failure is defined as greater than $50 \%$ change in any of the following parameters, $\mathrm{I}_{\mathrm{CBO}}, \mathrm{I}_{\mathrm{EBO}}, \mathrm{H}_{\mathrm{FE}}\left(10 \mathrm{~mA} \mathrm{I} \mathrm{I}_{\mathrm{C}}\right), \mathrm{V}_{\mathrm{CE}}$ (SAT) $\mathrm{V}_{\mathrm{BE}(\mathrm{SAT})}, \mathrm{V}_{\mathrm{CE}(\mathrm{SUS})}$ or $\mathrm{H}_{\mathrm{FE}}\left(50 \mu \mathrm{A} \mathrm{I}_{\mathrm{C}}\right)$. The plots clearly show the gettering properties of the glass.

\subsection{Ion Barrier Properties of Glassivation}

The previous experiment was repeated except that the glassivated sample was contaminated after glass deposition. A failure versus time graph is shown in Figure 3 for this experiment. Again the ion barrier characteristics are clearly shown by the comparison of the plots.

A second experiment was performed using an

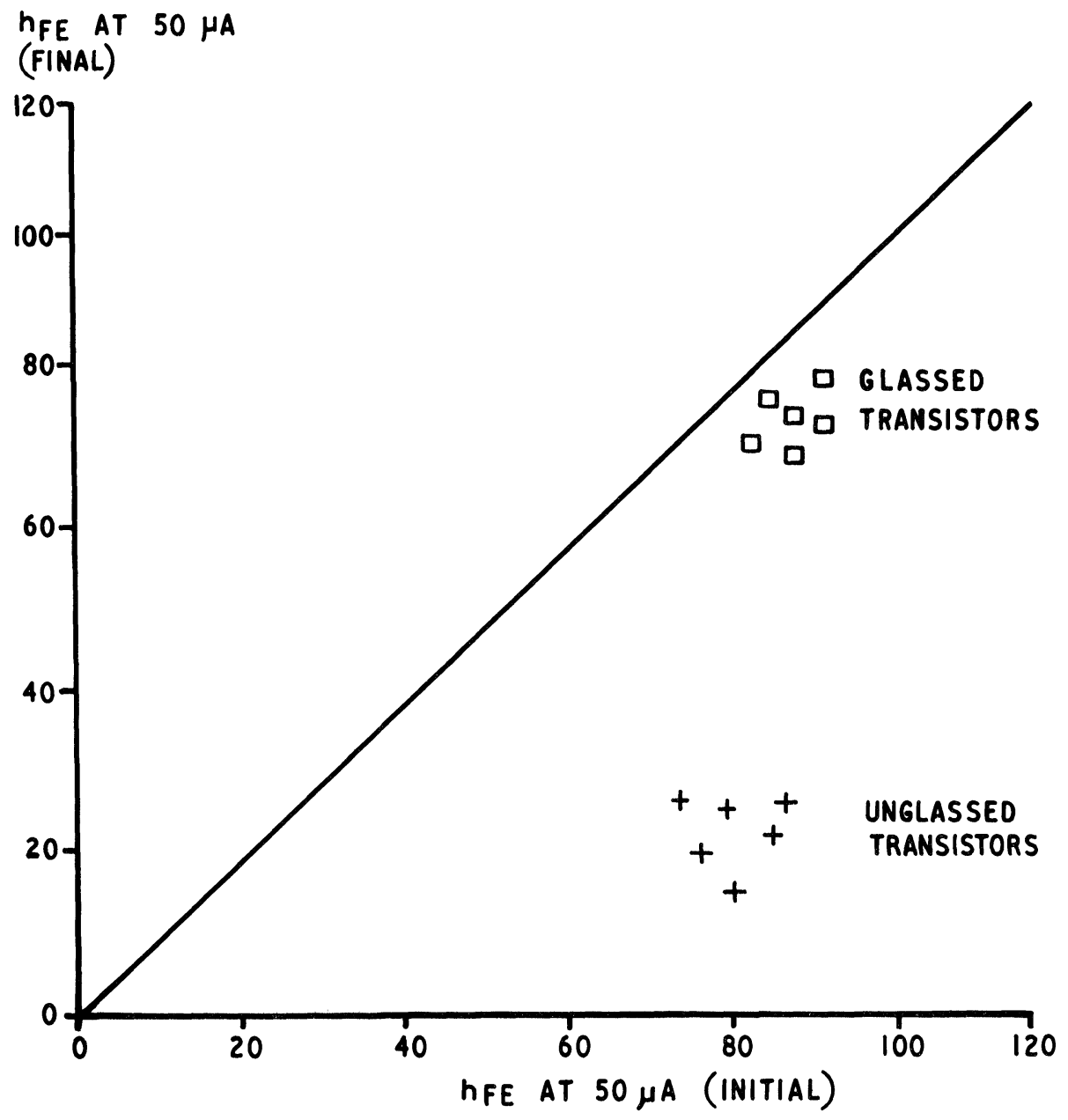

FIGURE 4 Graph of change in $\mathrm{h}_{\mathrm{FE}}$ for glassed and unglassed transistors in epoxy resin. 
epoxy encapsulant. The epoxy was known to polarise at temperatures in excess of $150^{\circ} \mathrm{C}$. Consequently if the device was stressed electrically under the same conditions as the previous experiment the dipoles when cooled down would be frozen in alignment with the applied electric fields. This would tend to cause instabilities in the encapsulated transistor. Figure 4 shows the stability of $50 \mu \mathrm{A} \mathrm{H} \mathrm{H}_{\mathrm{FE}}$ of the glassed and non-glassed chip in the epoxy resin. The glassivated devices are clearly more stable than the control devices. The thickness of the glass is sufficient to attenuate the dipole electric field at the $\mathrm{SiO}_{2} / \mathrm{Si}$ interface caused by the epoxy resin.

As a final test some glassivated BC107B transistors were subjected to the following tests. a) $230^{\circ} \mathrm{C} .160 \mathrm{hrs} \cdot \mathrm{V}_{\mathrm{CB}}=38 \mathrm{~V} \cdot \mathrm{V}_{\mathrm{EB}}=4 \mathrm{~V}$.

b) $25^{\circ} \mathrm{C} .160 \mathrm{hrs}$. $\mathrm{V}_{\mathrm{CE}}=40 \mathrm{~V} \cdot \mathrm{I}_{\mathrm{C}}=7 \mathrm{~mA}$ (i.e. $\mathrm{Tj} 230^{\circ} \mathrm{C}$ ).

c) Thermocycle $-40^{\circ} \mathrm{C}$ to $+100^{\circ} \mathrm{C}$.

A plot of $\mathrm{H}_{\mathrm{FE}}$ at $2 \mathrm{~mA}$ is shown in Figure 5 at the various test stages. It shows that the transistors remain stable throughout rigorous environmental testing.

\subsection{Toughness Evaluation}

A simple piece of equipment was designed and manufactured as shown in Figure 6. The sample would be placed on the block and the arm released by turning the cam so that the diamond point would

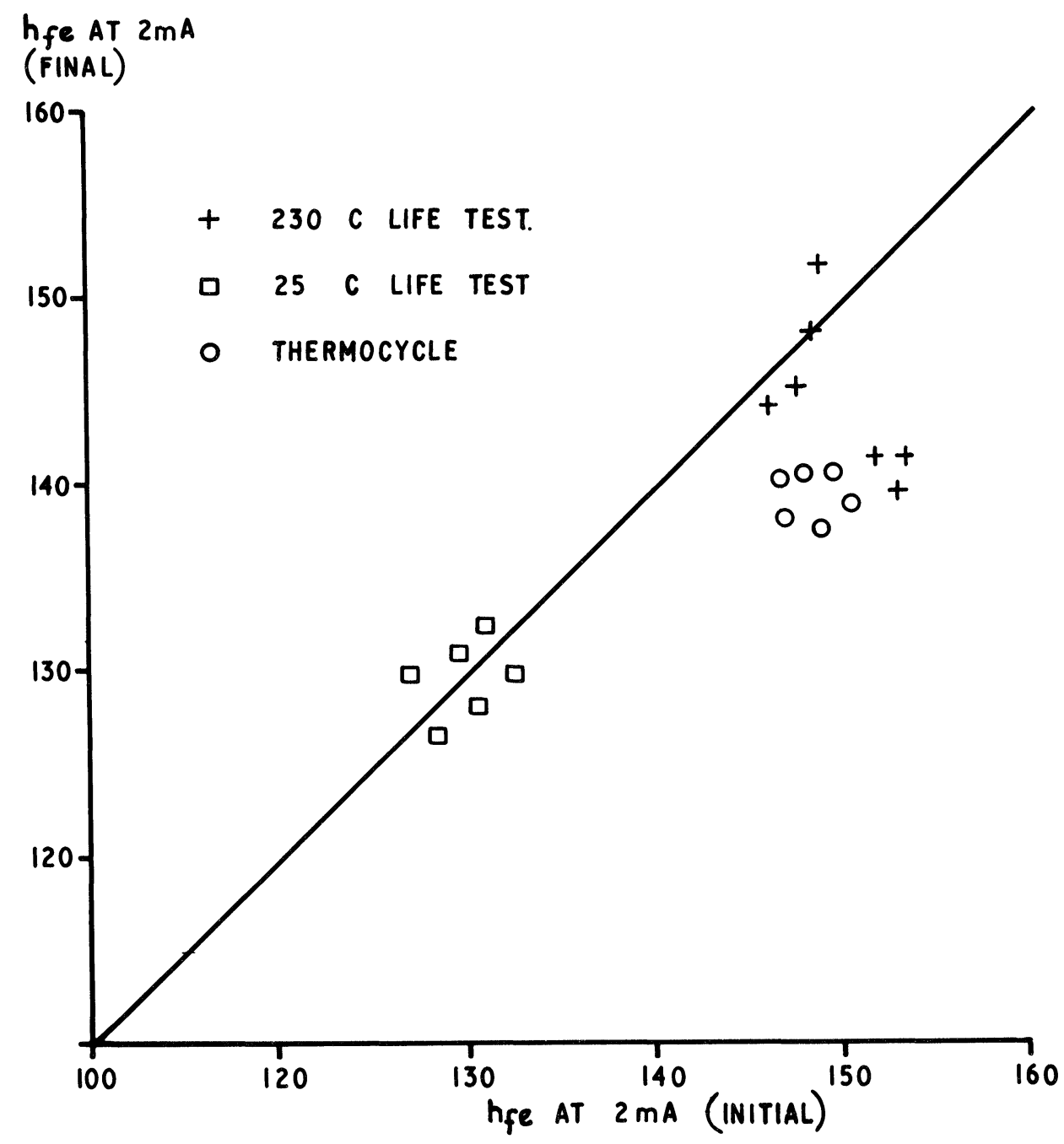

FIGURE 5 Graph of change in gain for glassivated ZTX 107B transistors in BS9000 test. 


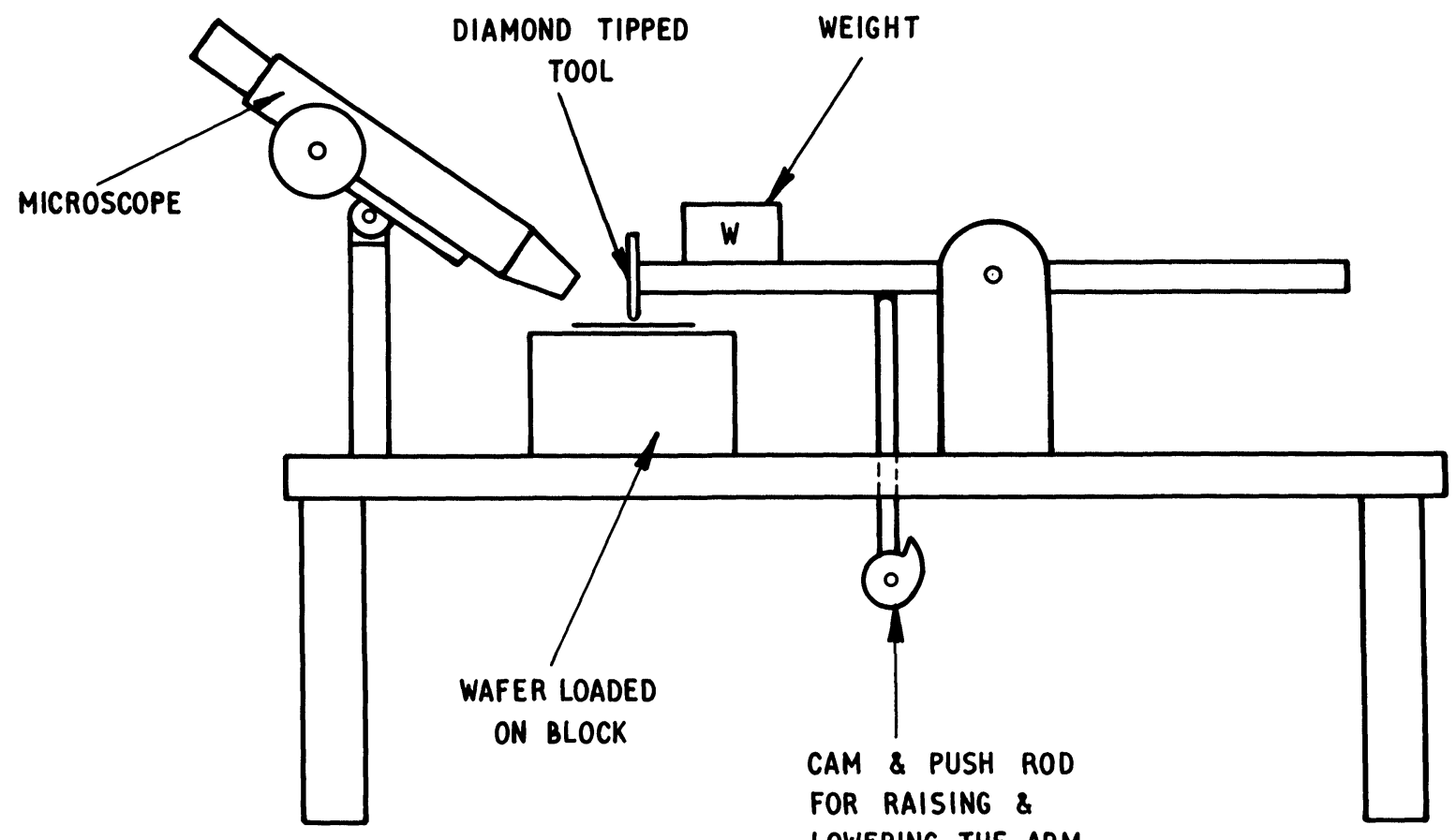

LOWERING THE ARM

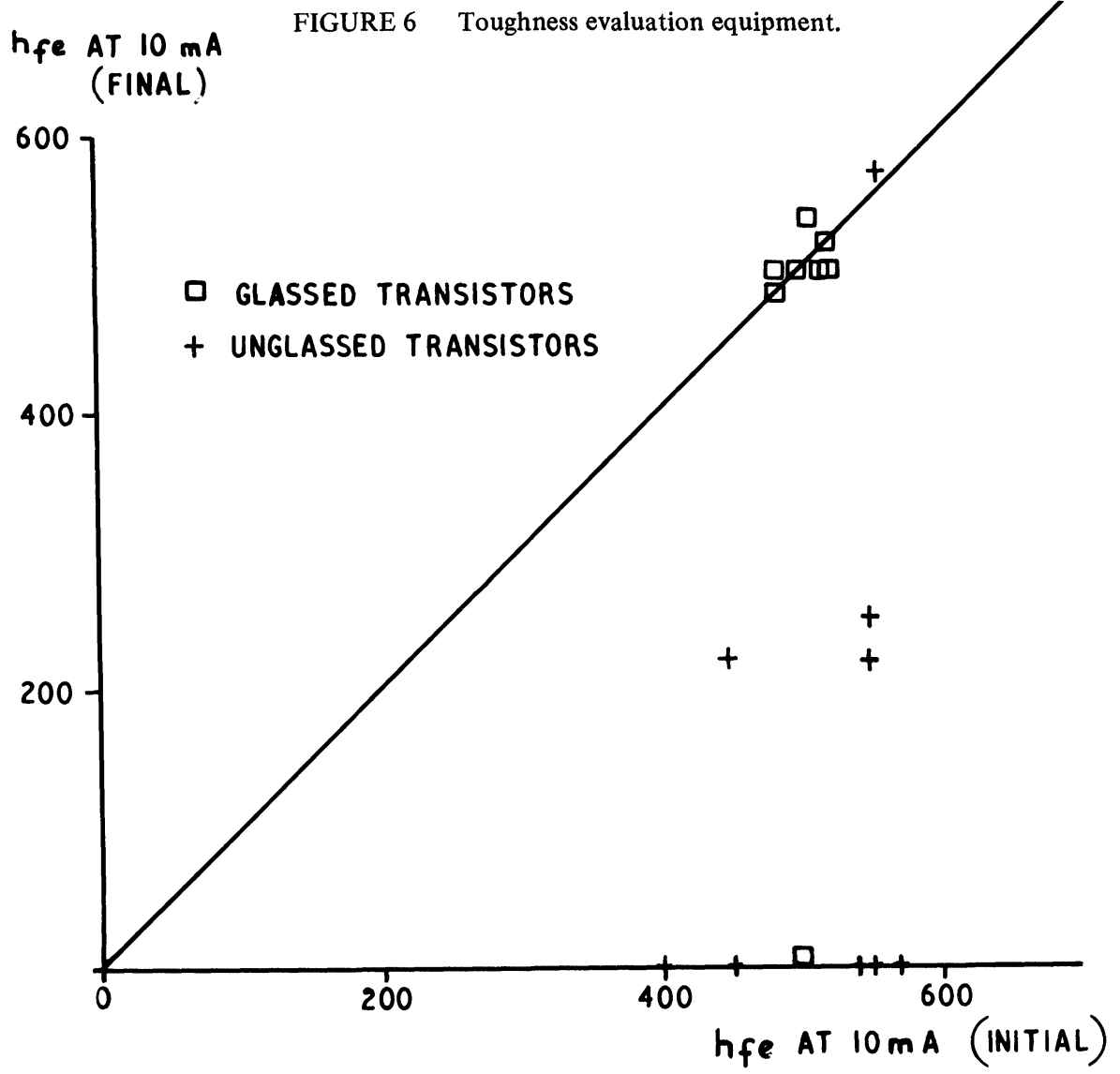

FIGURE 7 Graph of change $h_{f e}$ for glassed and unglassed transistors in cerpak. 
TABLE I

Change in 10mA HFE for glassed and unglassed transistors assembled in cerpak

Glass Passivated

\begin{tabular}{lllllllllllllll}
\hline Package & & $1 G$ & $2 G$ & $3 G$ & $4 G$ & $5 G$ & $6 G$ & $7 G$ & $8 G$ & $9 G$ & $10 G$ & $11 G$ & $12 G$ \\
\hline T1 Hfe @ & Initial & 510 & 500 & 490 & 500 & 500 & 480 & 480 & 500 & 500 & 500 & 500 & 490 \\
10mA 5V. & final & 500 & 530 & 530 & 510 & 500 & 490 & - & 500 & 540 & 520 & 530 & 520 \\
& Initial & 490 & 520 & 500 & 480 & 490 & 490 & 500 & 510 & 490 & 480 & 480 & 490 \\
T2 Hfe@ & final & 500 & 520 & 530 & 540 & 550 & 500 & - & 530 & 510 & 510 & 510 & 520 \\
10mA 5V. & Initial & 500 & 490 & 500 & 510 & 500 & 500 & 490 & 490 & 480 & 490 & 490 & 480 \\
T3 Hfe @ & final & - & 490 & 520 & 510 & 530 & 510 & - & 520 & 550 & 510 & 510 & 510 \\
10mA 5V. & Initial & 510 & 500 & 500 & 490 & 500 & 510 & 500 & 490 & 490 & 480 & 500 & 490 \\
& final & 500 & 500 & 520 & 520 & 510 & 520 & - & 510 & - & 520 & 540 & 510 \\
T4 Hfe@ & & & & & & & & & & & & & &
\end{tabular}

Non Glass Passivated

\begin{tabular}{lllllllllllllll}
\hline Package & & 13 & 14 & 15 & 16 & 17 & 18 & 19 & 20 & 21 & 22 & 23 & 24 \\
\hline T1 Hfe @ & Initial & 550 & 570 & 520 & 550 & 570 & 550 & 570 & 590 & 590 & 550 & 570 & 570 \\
10mA 5V. & final & 220 & - & 650 & 580 & 310 & - & 600 & - & 600 & 470 & - & 560 \\
& Initial & 550 & 400 & 540 & 570 & 520 & 540 & 580 & 540 & 550 & 560 & 580 & 560 \\
T2 Hfe @ & final & 250 & - & 530 & 250 & 230 & 430 & 560 & 280 & 340 & - & - & - \\
10mA 5V. & Initial & 540 & 450 & 560 & 560 & 570 & 560 & 570 & 560 & 560 & 550 & 570 & 600 \\
T3 Hfe @ & final & - & 220 & - & - & - & - & 400 & - & 480 & 540 & - & - \\
10mA 5V. & Initial & 550 & 560 & 550 & 570 & 560 & 540 & 300 & 560 & 530 & 590 & 570 & 600 \\
T4 Hfe @ & final & - & 570 & - & - & - & - & - & - & - & - & - & - \\
$10 \mathrm{~mA} \mathrm{5V.}$ & & & & & & & & & & & & & &
\end{tabular}

be dropped from a consistent height (i.e. $2 \mathrm{~cm}$ ) onto the sample. Additional weights would be placed on the arm in order to increase the force applied. This trial would test the resistance of the glass to vertically directed damaging forces. The sample itself consisted of a 1.5 micron Aluminium layer on a silicon substrate covered with a 2 micron thick layer of glass. Failure was adjudged to have occured when small cracks just emanated from the point of impact.

Horizontally directed damaging forces were investigated by slowly lowering the point on to the glass and moving the sample in a horizontal direction. The weight of the arm was gradually increased and the critical weight was reached when the point broke through the glass layer and exposed aluminium beneath. Results showed that the glass consistently failed in the vertical mode with a force of $4 \mathrm{gms}$ and in the horizontal mode with a force of $12 \mathrm{gms}$.

\subsection{Assembly Evaluation}

To evaluate the ability of glassivated chips to withstand various time temperature combinations encountered in typical assembly operations, a sample experiment was performed. 48 glassivated and 48 non-glassivated control chips from the same diffused wafer were measured for $10 \mathrm{~mA}$ gain. The devices were $\mathrm{Si} \mathrm{Au}$ eutectically attached to a 4 position flatpack frame to make up 12 flatpack arrays each for the glassivated and control batches. The frames were then glass fritted on to a ceramic base, ultrasonic Aluminium wire bonded the emitter and base to the frame and then hermetically glass sealed with a ceramic cap. The highest temperature to which the devices were subjected was $500^{\circ} \mathrm{C}$ during the sealing operation. The times were typical for each operation whilst noting that one transistor chip will remain at 
temperature whilst the other three devices are being alloyed and bonded.

The initial $10 \mathrm{~mA}$ gains were measured on the transistors in chip form and then subsequently remeasured after subjecting them to the previously described assembly procedure. The results are shown in Table I. Devices from packages 1G, 2G and 13, and 14 respectively were plotted on an $\mathrm{H}_{\mathrm{FE}}$ change graph to illustrate the results comparatively. The package yield (i.e. 4 good transistors/package) for glassivated chips was $75 \%$. The chip yield was $88 \%$. In comparison the package yield for nonglassivated chips was zero. The chip yield was $17 \%$. The criteria of failure was a greater than $20 \%$ shift in $\mathrm{H}_{\mathrm{FE}}$ (i.e. standard for BS9000 or C.E.C.C. rules). The results clearly indicate the greatly increased robustness of glassivated chips. (See Figure 7).

\section{CONCLUSIONS}

It has been demonstrated that deposition and firing of the glass layer on a silicon bipolar transistor effectively neutralises any ionic contamination which may have been present prior to deposition. The glass layer has been shown to be impervious to ionic impurities and to be of sufficient thickness to eliminate any effect due to polarising encapsulants etc.

The toughness experiment indicated that glassivated devices could withstand the normal tweezer handling experienced in assembly operations and it effectively protects the underlying aluminium layer from scuffing. Finally the assembly evaluation indicates the degree to which the glassivated devices will withstand long and high, time temperature combinations well in excess of those at which standard nonglassivated material exhibits parameter degradation. 

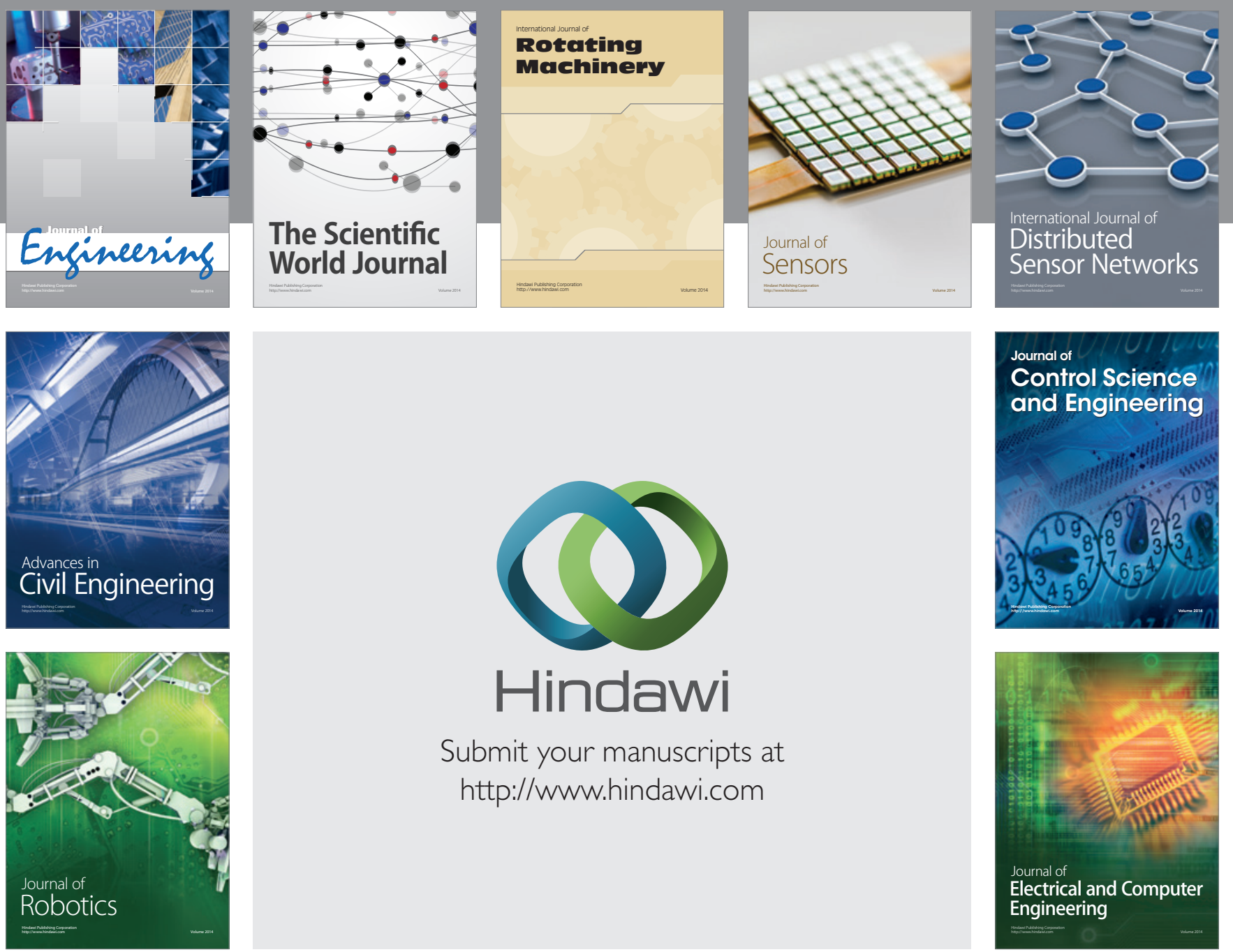

Submit your manuscripts at

http://www.hindawi.com
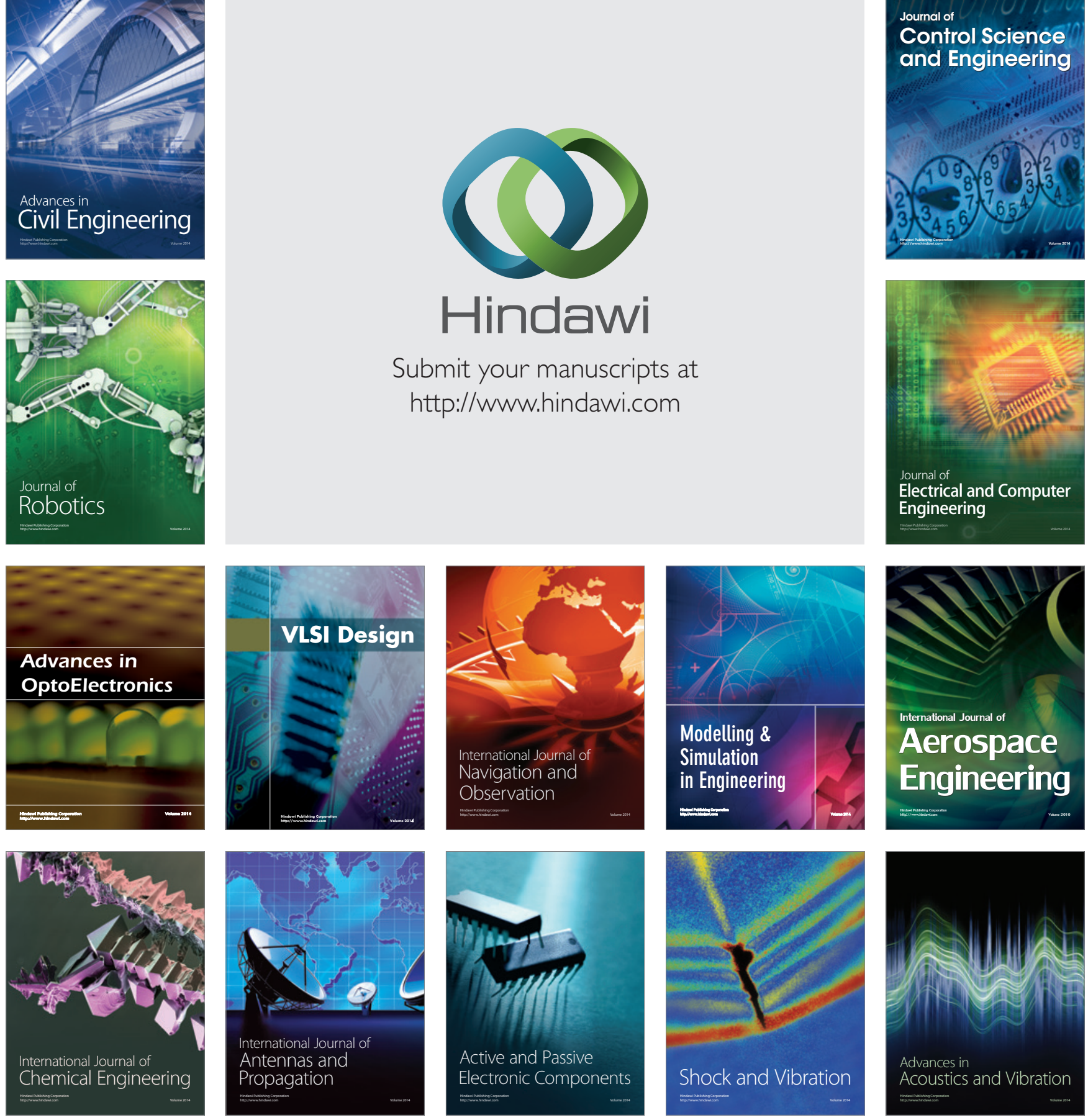